\title{
Trap levels in layered semiconductor $\mathrm{Ga}_{2} \mathrm{SeS}$
}

\author{
A. Aydinli ${ }^{a, *}$, N.M. Gasanly ${ }^{\mathrm{b}}$, S. Aytekin ${ }^{\mathrm{a}}$ \\ ${ }^{a}$ Department of Physics, Bilkent University, 06533 Ankara, Turkey \\ ${ }^{\mathrm{b}}$ Department of Physics, Middle East Technical University, 06531 Ankara, Turkey
}

Received 23 July 2004; accepted 23 August 2004 by P. Wachter

Available online 2 November 2004

\begin{abstract}
Trap levels in nominally undoped $\mathrm{Ga}_{2} \mathrm{SeS}$ layered crystals have been characterized by thermally stimulated current (TSC) measurements. During the measurements, current was allowed to flow along the $c$-axis of the crystals in the temperature range of 10-300 K. Two distinct TSC peaks were observed in the spectra, deconvolution of which yielded three peaks. The results are analyzed by curve fitting, peak shape and initial rise methods. They all seem to be in good agreement with each other. The activation energies of three trapping centers in $\mathrm{Ga}_{2} \mathrm{SeS}$ are found to be 72,100 and $150 \mathrm{meV}$. The capture cross section of these traps are $6.7 \times 10^{-23}, 1.8 \times 10^{-23}$ and $2.8 \times 10^{-22} \mathrm{~cm}^{2}$ with concentrations of $1.3 \times 10^{12}, 5.4 \times 10^{12}$ and $4.2 \times 10^{12} \mathrm{~cm}^{-3}$, respectively.
\end{abstract}

(c) 2004 Elsevier Ltd. All rights reserved.

PACS: 77.20.Jv; 71.55.-I; 72.80.Jc

Keywords: A. Semiconductors; A. Chalcogenides; C. Defects; D. Electrical properties

\section{Introduction}

Gallium selenide-sulfide $\left(\mathrm{Ga}_{2} \mathrm{SeS}\right)$ is a ternary layered semiconductor of the $\mathrm{A}^{\mathrm{III}} \mathrm{B}^{\mathrm{VI}}$ family. This material displays the properties of its layered parent compounds, GaSe and $\mathrm{GaS}$, which form the mixed crystals. Each layer has four atomic planes with the sequence $\mathrm{S}(\mathrm{Se})-\mathrm{Ga}-\mathrm{Ga}-\mathrm{S}(\mathrm{Se})$. The interlayer bonding is van der Waals like and weak, whereas the intralayer bonding is covalent and strong. The indirect band gap of both GaSe and $\mathrm{GaS}$ is reflected in the energy band diagram of $\mathrm{Ga}_{2} \mathrm{SeS}$ crystal with energy gap of 2.33 and $2.27 \mathrm{eV}$ at 10 and $300 \mathrm{~K}$, respectively [1,2]. $\mathrm{Ga}_{2} \mathrm{SeS}$ may be a promising semiconductor for applications in optoelectronic devices such as near-blue emitting and detecting devices [3-5]. The anisotropic nature of the crystal may also

\footnotetext{
* Corresponding author. Tel.: +90-312-290-1579; fax:+90-312266-4579.

E-mail address: aydinli@bilkent.edu.tr (A. Aydinli).
}

allow the use of $\mathrm{Ga}_{2} \mathrm{SeS}$ in polarization selective waveguide devices such as polarization splitters, modulators, etc. However, the presence of impurity and/or defect centers in any semiconductor crystal is one of the determining factors in the eventual device performance. In the case of light emitting devices, defect levels may act as non-radiative recombination centers (traps) lowering internal quantum efficiency, while in the case of electronic devices, defect centers may decrease carrier mobility leading to degradation of the frequency response of the devices. Therefore, it is important to get detailed information on energetic and kinetic parameters of trapping centers of $\mathrm{Ga}_{2} \mathrm{SeS}$ crystals.

There are several methods to determine the properties of trap centers in semiconductors. Among them, thermally stimulated current (TSC) measurements are distinguished by the ease with which the experiment is performed and the detailed information it provides on trap states [6,7]. The experimental procedure of TSC measurement consists of the following: the samples are illuminated with a suitable light source at low temperature, during which the traps are filled 
by band-to-band excitation of carriers. The trapped charge carriers are thermally released to the conduction or the valence band during heating. Thermally released carriers give rise to a transient increase in the conductivity of the sample. The temperature is raised at a constant rate for the sake of convenience of the analysis. TSC curves for a single trap show a slightly asymmetry with a fairly sharp maximum at a temperature which is determined by the activation energy, capture cross section and concentration of the traps, and the heating rate. If more than one type of trap is present, current through the sample due to thermal stimulation may be expected to show several maxima.

There is only a brief report on the TSC study of defect traps in $\mathrm{Ga}_{2} \mathrm{SeS}$ crystals, grown using the iodine transport method, in the temperature range 80-300 K [8]. Two trap levels with activation energy of 155 and $400 \mathrm{meV}$ have been found in the band gap. In our previous paper [9], we presented the results of photoluminescence (PL) investigation of $\mathrm{Ga}_{2} \mathrm{SeS}$ crystals in the $565-860 \mathrm{~nm}$ wavelength region and in the $15-170 \mathrm{~K}$ temperature range. Two donor levels located at 29 and $40 \mathrm{meV}$ were determined from the temperature dependence of PL intensity.

The purpose of the present work is to obtain further information concerning the deep and shallow defects in undoped $\mathrm{Ga}_{2} \mathrm{SeS}$ crystals that may arise due to structural defects and/or presence of unintentional impurities using the well-established technique of TSC measurements. In contrast with previous TSC measurements on $\mathrm{Ga}_{2} \mathrm{SeS}$ crystals, we for the first time employ a wide temperature range of $10-300 \mathrm{~K}$. The measurements in the temperature range below $80 \mathrm{~K}$ allow us to check for the possibility of shallow trap states. We report on the activation energy, capture cross section, attempt-to-escape frequency and concentration of the traps in $\mathrm{Ga}_{2} \mathrm{SeS}$ crystals.

\section{Experimental procedure}

$\mathrm{Ga}_{2} \mathrm{SeS}$ polycrystals were synthesized from the elements with high purity (at least $99.999 \%$ ) taken in stochiometric proportions. $\mathrm{Ga}_{2} \mathrm{SeS}$ single crystals were grown by the modified Bridgman method. $\mathrm{Ga}_{2} \mathrm{SeS}$ crystallizes in the hexagonal unit cell with lattice parameters of $a=0.3671 \mathrm{~nm}$ and $c=1.5719 \mathrm{~nm}$ as obtained from X-ray diffraction data. Crystals suitable for TSC measurements were obtained by easy cleavage of an ingot along the layers, which is perpendicular to the $c$-axis. Typical sample dimensions were $7.2 \times 3.0 \times 1.2 \mathrm{~mm}^{3}$. The room temperature conductivity, electron concentration, mobility and effective mass were $1.9 \times 10^{-5}(\Omega \mathrm{cm})^{-1}, 1.5 \times 10^{12} \mathrm{~cm}^{-3}, 80 \mathrm{~cm}^{2} \mathrm{~V}^{-1} \mathrm{~s}^{-1}$ and $0.41 \mathrm{~m}_{0}$, respectively [10]. The $488.0 \mathrm{~nm}$ line of the $\mathrm{Ar}^{+}$laser was used as the excitation light source. A closed cycle helium cryostat was used to cool the samples down to $10 \mathrm{~K}$. The temperature of the cryostat was controlled to within $\pm 0.5 \mathrm{~K}$.

The electrodes were deposited by evaporating gold under high vacuum, on both sides of the crystal surfaces to arrange sandwich geometry. Care was taken to mask the edges of the crystals to avoid electrical shorting of the samples. Thickness of the gold electrodes was about $100 \mathrm{~nm}$ on back side and $10 \mathrm{~nm}$ on front side, the latter corresponding to higher transmittance of the incident light. The sample was mounted on the cold finger of a cryostat with conducting silver paste and dried at room temperature. The backside was grounded through the sample holder. A thin isolated gold wire was attached to the front side electrode by a small droplet of silver paste. The $I-V$ characteristics were checked to be symmetric with respect to the polarity. The thermally stimulated currents, measured by a 'Keithley 619' electrometer, and temperature data were stored in a personal computer.

In a typical experiment, samples are cooled down to $T=$ $10 \mathrm{~K}$ and kept at this temperature for $10 \mathrm{~min}$. Then the sample surface is illuminated through the semitransparent front electrode for a fixed period of time $(10 \mathrm{~min})$, at an applied bias voltage of $V_{1}=10 \mathrm{~V}$. After the light is turned off, the photoconductivity signal is allowed to decay for $15 \mathrm{~min}$. Just before the heating process a bias voltage of $V_{2}=50 \mathrm{~V}$ was applied to the sample. The samples are then heated at a constant rate of $\beta=0.18 \mathrm{~K} / \mathrm{s}$ from 10 to $300 \mathrm{~K}$.

\section{Results and discussion}

Fig. 1 shows a typical TSC curve for $\mathrm{Ga}_{2} \mathrm{SeS}$ crystal measured with heating rate of $\beta=0.18 \mathrm{~K} / \mathrm{s}$. Three peaks are determined at temperatures $83.2,117.8$ and $136.8 \mathrm{~K}$, after deconvolution of the TSC peaks by a curve fitting technique. TSC spectra in the high-temperature region $(T>160 \mathrm{~K})$ could not be measured due to strong increase in dark

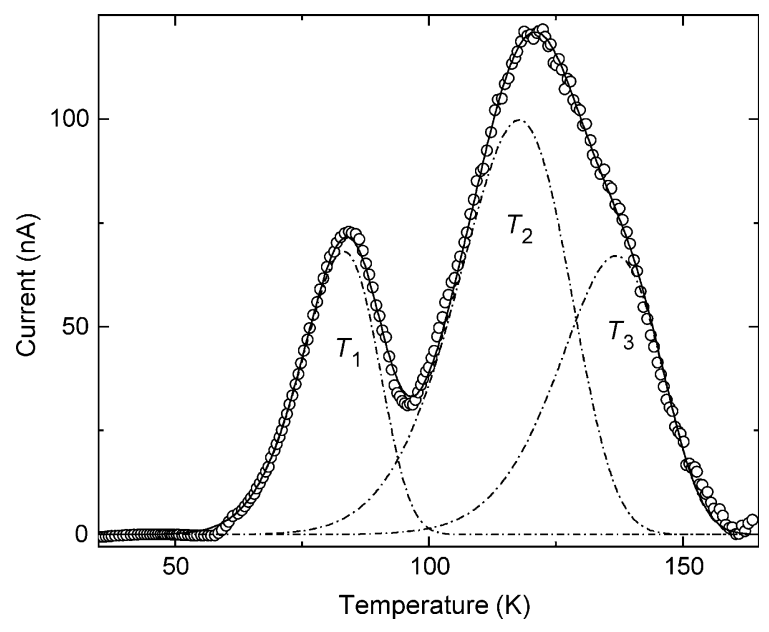

Fig. 1. Experimental TSC spectrum of $\mathrm{Ga}_{2} \mathrm{SeS}$ crystal and decomposition of this spectrum into three separate peaks. Open circles are experimental data. Dashed curves represent decomposed peaks. Solid curve shows total fit to the experimental data. 


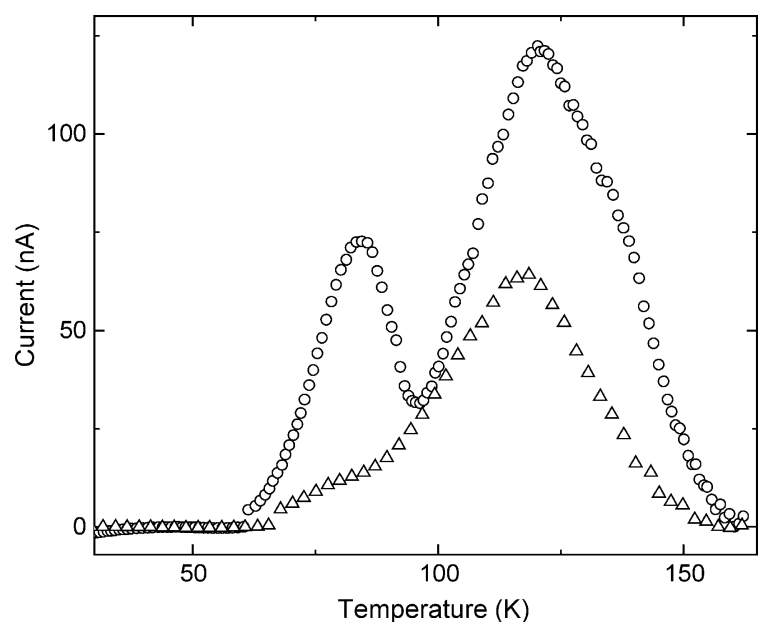

Fig. 2. Typical TSC curves of $\mathrm{Ga}_{2} \mathrm{SeS}$ crystal under opposite bias voltage. Open circles and triangles represent the experimental data obtained at illumination of negative and positive contacts, respectively.

conductivity in this region. In Fig. 2, we show the TSC spectra of $\mathrm{Ga}_{2} \mathrm{SeS}$ both for forward and reverse bias conditions. As can clearly be observed, the TSC is significantly larger for the forward bias condition (illumination of negative contact). In fact, for the first peak with the lowest temperature and activation energy, there is negligible TSC under reverse bias condition. Therefore, this peak can be attributed to electron traps. As for the high-temperature peaks, the TSC under reverse bias is approximately 50\% that of the forward biased case which does not allow us to make a definitive identification of the trapped carrier type.

Activation energies of the traps are determined using three different methods: curve fitting, peak shape and initial rise methods.

\subsection{Curve fitting method}

Relative magnitudes of capture cross sections $S_{\mathrm{t}}$ and $S_{\mathrm{r}}$ of the trapping and recombination centers, respectively, play an important role in the analysis of the TSC data. For monomolecular process $S_{\mathrm{t}} \ll S_{\mathrm{r}}$, hence slow retrapping occurs. In the case of $S_{\mathrm{t}}=S_{\mathrm{r}}$, the process is bimolecular, and for the case $S_{\mathrm{t}} \gg S_{\mathrm{r}}$ fast retrapping occurs. We have tried all possibilities and found that assumption of monomolecular process gives the best results for this crystal. Under monomolecular conditions (i.e. slow retrapping) the TSC curve of a discrete set of traps with a trapping level $E_{t}$ below the conduction band is described by the equation [11]

$\sigma=n_{\mathrm{t} 0} \tau e \mu \nu \exp \left[-E_{\mathrm{t}} / k T-\int_{T_{0}}^{T}(v / \beta) \exp \left(-E_{\mathrm{t}} / k T\right) \mathrm{d} T\right]$.

Here, $\sigma$ is the thermally stimulated conductivity, $n_{t 0}$ the initial density of filled traps, $\tau$ the lifetime of a free electron, $\mu$ the electron mobility, $\beta$ the heating rate and $T_{0}$ the temperature from which heating begins following the filling of traps. $\nu$ is the attempt-to-escape frequency of a trapped electron:

$\nu=N_{\mathrm{c}} v_{\mathrm{th}} S_{\mathrm{t}}$,

where $N_{\mathrm{c}}=2\left(2 \pi m_{e}^{*} k T / h^{2}\right)^{3 / 2}$ is the effective density of states in the conduction band and $u$ th is the electron thermal velocity. If it is assumed that $v$ is independent on $T$ and over the temperature span of TSC curve, the variation of $\mu$ and $\tau$ with $T$ can be ignored, Eq. (1) can be rewritten approximately as:

$\sigma=A \exp \left[-t-B \exp (-t) t^{-2}\right]$,

where $t=E_{t} / k T$, and $\mathrm{A}$ and $\mathrm{B}$ are constants: $A=n_{t o} \tau e \mu \nu$ and $B=v E_{t} \beta k$.

If Eq. (3) is differentiated and equated to zero to find the maximum of the curve, which occurs when $t=t_{\mathrm{m}}=E_{\mathrm{t} /} k T_{\mathrm{m}}$, then

$B=\exp \left(t_{\mathrm{m}}\right) t_{\mathrm{m}}^{3} /\left(t_{\mathrm{m}}+2\right)$.

In TSC spectra of $\mathrm{Ga}_{2} \mathrm{SeS}$, we obtained three peaks, $T_{1}$, $T_{2}$ and $T_{3}$, with $T_{2}$ and $T_{3}$ overlapping each other. Therefore the following fit function was used

$\sigma_{\text {total }}=\sum \sigma_{\mathrm{m}}$,

where $m$ denotes the number of traps involved in calculation.

Good agreement between the experimental TSC curve and theoretical one, computed with the assumption of slow retrapping, suggests that retrapping does not occur for the trapping centers studied (Fig. 1). The procedure described above allowed us to obtain $E_{\mathrm{t}}$ and $T_{\mathrm{m}}$ for each peak directly from the fit. After finding $E_{\mathrm{t}}$ and $t_{\mathrm{m}}$, we can determine $v=$ $B \beta k / E_{\mathrm{t}}$. Then we calculated $S_{\mathrm{t}}$ from Eq. (2) for all three peaks. The obtained values of activation energy, capture cross section and attempt-to-escape frequency for peaks $T_{1}$, $T_{2}$ and $T_{3}$ are shown in Table 1.

\subsection{Peak shape method}

The shape of the TSC peaks also yields information on the activation energy of the traps [11]. Three parameters: $\tau=T_{\mathrm{m}}-T_{\mathrm{l}}, \delta=T_{\mathrm{h}}-T_{\mathrm{m}}$ and $w=T_{\mathrm{h}}-T_{\mathrm{l}}$ of a given TSC peak, where $T_{\mathrm{m}}$ is the temperature corresponding to the maximum current, $T_{1}$ and $T_{\mathrm{h}}$ are the low and high halfintensity temperatures, respectively, are sufficient to obtain the activation energy of a given trap

$$
\begin{aligned}
& E_{\tau}=\left\{\left[1.51+3.0\left(\mu_{\mathrm{g}}-0.42\right)\right] k T_{\mathrm{m}}^{2} / \tau\right\}-\left[1.58+4.2\left(\mu_{\mathrm{g}}\right.\right. \\
&-0.42)] 2 k T_{\mathrm{m}}, \\
& E_{\delta}=\left\{\left[0.976+7.3\left(\mu_{\mathrm{g}}-0.42\right)\right] k T_{\mathrm{m}}^{2} / \delta,\right. \\
& E_{w}=\left\{\left[2.52+10.2\left(\mu_{\mathrm{g}}-0.42\right)\right] k T_{\mathrm{m}}^{2} / w\right\}-2 k T_{\mathrm{m}} .
\end{aligned}
$$


Table 1

The activation energy $\left(E_{\mathrm{t}}\right)$, capture cross section $\left(S_{\mathrm{t}}\right)$, attempt-to-escape frequency $(\nu)$ and concentration $\left(N_{\mathrm{t}}\right)$ of traps for three TSC peaks of $\mathrm{Ga}_{2} \mathrm{SeS}$ crystal

\begin{tabular}{|c|c|c|c|c|c|c|c|}
\hline \multirow[t]{2}{*}{ Peak } & \multirow[t]{2}{*}{$T_{\mathrm{m}}(\mathrm{K})$} & \multicolumn{3}{|l|}{$E_{\mathrm{t}}(\mathrm{meV})$} & \multirow[t]{2}{*}{$S_{\mathrm{t}}\left(\mathrm{cm}^{2}\right)$} & \multirow[t]{2}{*}{$\nu\left(\mathrm{s}^{-1}\right)$} & \multirow[t]{2}{*}{$N_{\mathrm{t}}\left(\mathrm{cm}^{-3}\right)$} \\
\hline & & $\begin{array}{l}\text { Curve fitting } \\
\text { method }\end{array}$ & $\begin{array}{l}\text { Peak shape } \\
\text { method }\end{array}$ & $\begin{array}{l}\text { Initial rise } \\
\text { method }\end{array}$ & & & \\
\hline$\overline{T_{1}}$ & 83.2 & 72 & 81 & 68 & $6.7 \times 10^{-23}$ & $4.0 \times 10^{2}$ & $1.3 \times 10^{12}$ \\
\hline$T_{2}$ & 117.8 & 100 & 107 & 95 & $1.8 \times 10^{-23}$ & $2.1 \times 10^{2}$ & $5.4 \times 10^{12}$ \\
\hline$T_{3}$ & 136.8 & 150 & 159 & 143 & $2.8 \times 10^{-22}$ & $4.4 \times 10^{3}$ & $4.2 \times 10^{12}$ \\
\hline
\end{tabular}

Chen and Kirsh [11] have predicted the values of $\mu_{\mathrm{g}}(=$ $\delta / w)$ as 0.42 for first order and 0.52 for second order kinetics. Values of $\mu_{\mathrm{g}}$ obtained for our decomposed peaks $T_{1}, T_{2}$ and $T_{3}$ were found to be $0.45,0.44$ and 0.43 , respectively. Therefore, we consider the observed TSC peaks as first order. The averaged values of calculated activation energies $E_{\tau}, E_{\delta}$ and $E_{w}$ for three peaks are reported in Table 1.

\subsection{Initial rise method}

Valid for all types of recombination kinetics, the initial rise method [11] is based on the assumption that, when the traps begin to empty as the temperature is increased, the intensity of TSC is proportional to $C \times \exp \left(-E_{\mathrm{t}} / k T\right)$, where the constant $C$ depends on the number of initially full traps and the probabilities of the transitions involved. Thus, a plot of the logarithm of the current intensity against $1 / T$ should yield a straight line with a slope of $\left(-E_{\mathrm{t}} / k\right)$, as shown in Fig. 3 . The activation energies of the traps calculated by this procedure are found to be 68,95 and $143 \mathrm{meV}$ for peaks $T_{1}$, $T_{2}$ and $T_{3}$, respectively (Table 1 ).

At this point, it is worthwhile to compare present results with those obtained in our previous studies of $\mathrm{Ga}_{2} \mathrm{SeS}$ crystals using PL spectroscopy, which yielded two levels at 29 and $40 \mathrm{meV}$ [9]. These levels were obtained from the temperature dependence of the PL intensity. From the

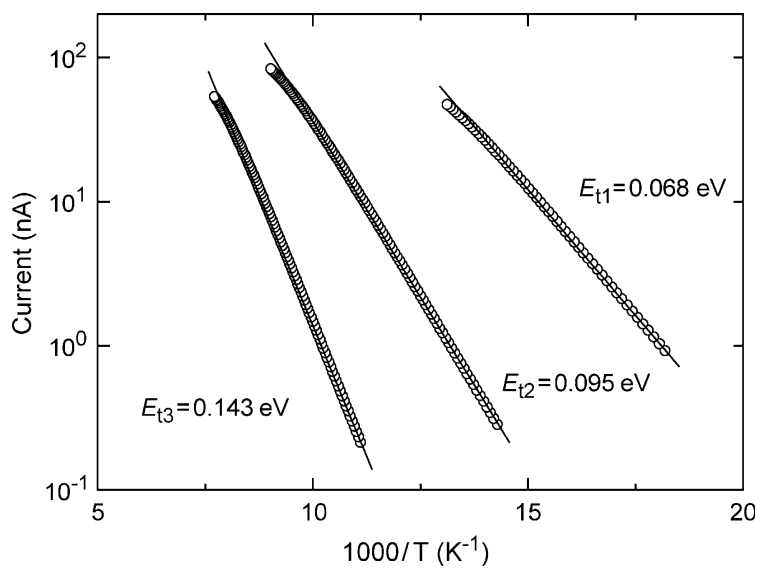

Fig. 3. TSC vs. 1000/T for all three peaks in TSC spectrum of $\mathrm{Ga}_{2} \mathrm{SeS}$ crystal. Open circles and solid lines represent experimental data and theoretical fits using initial rise method, respectively. present results of TSC, we observe three trap levels at 72, 100 and $150 \mathrm{meV}$. It is clear that the PL levels observed at 29 and $40 \mathrm{meV}$ are not observed in the TSC experiments and the levels at 72, 100 and $150 \mathrm{meV}$ obtained from TSC measurements are not observed in the PL experiments. We suppose that the former two levels are recombination levels and the latter three levels are trapping levels, allowing for either PL emission or thermally stimulated current, respectively.

In the previous TSC work on $\mathrm{Ga}_{2} \mathrm{SeS}$ grown using the iodine assisted chemical transport method, trap levels with activation energies of 155 and $400 \mathrm{meV}$ have been found in the band gap of crystals [8]. In that work, the nature of the first level was not explained, while the second level was associated with the presence of iodine, incorporated during crystal growth. Considering that our samples were grown using the modified Bridgman method with no intentional doping or a carrier agent, it is not surprising that we did not observe the level at $400 \mathrm{meV}$, as in [8]. The only trap levels, which are close in energy, are located at 150 (present work) and $155 \mathrm{meV}$ [8]. Therefore, we may assign the obtained energies to the same level, which may originate from the presence of structural defects, independent of the crystal growing method.

\subsection{Trap concentration determination}

The concentration of the traps was estimated using the relation [12]

$N_{\mathrm{t}}=\frac{Q}{A L e G}$.

Here, $Q$ is the quantity of charge released during a TSC experiment and can be calculated from the area under the TSC peaks; $A$ and $L$ are the area and the thickness of the sample, respectively; $e$ is the electronic charge and $G$ is the photoconductivity gain. The latter parameter was evaluated from the ratio

$G=\frac{\tau}{\mathrm{t}_{\mathrm{tr}}}=\frac{\tau \mu V}{L^{2}}$

where $t_{\mathrm{tr}}$ is the electron transit time between the electrodes and $V$ is the applied voltage. Electron lifetimes measured from the decay of photocurrent were equal $5.0 \times 10^{-3}$, $2.5 \times 10^{-3}$ and $2.0 \times 10^{-3} \mathrm{~s}$ for the peaks $T_{1}, T_{2}$ and $T_{3}$, 
respectively. The corresponding values of photoconductivity gain were found to be 1390,695 , and 555 . The values of $N_{\mathrm{t}}$ obtained for three traps are presented in Table 1.

\section{Conclusions}

Three trapping levels at 72, 100 and $150 \mathrm{meV}$ have been detected in as-grown $\mathrm{Ga}_{2} \mathrm{SeS}$ layered crystals by the TSC technique. These levels in undoped layered crystals may be associated with the presence of stacking faults and/or unintentional impurities. The activation energies of the peaks, evaluated by the curve fitting method, and also calculated using the peak shape and initial rise methods from the isolated peaks, are in agreement with each other, within the accuracy of the method used. The capture cross section of the traps were calculated to be $6.7 \times 10^{-23}, 1.8 \times 10^{-23}$ and $2.8 \times 10^{-22} \mathrm{~cm}^{2}$. The concentration of the traps were estimated as $1.3 \times 10^{12}, 5.4 \times 10^{12}$ and $4.2 \times 10^{12} \mathrm{~cm}^{-3}$.

\section{Acknowledgements}

This work was supported by a Bilkent University Research grant (Code: Phys-03-02). We thank M. Goktepe and N.S. Yuksek for their help with the measurements.

\section{References}

[1] C. Manfredotti, A. Rizzo, A. Bufo, V.L. Cardetta, Phys. Status Solidi A 30 (1975) 375.

[2] A. Mercier, J.P. Voitchovsky, J. Phys. Chem. Sol. 36 (1975) 1411.

[3] T. Aono, K. Kase, A. Kinoshita, J. Appl. Phys. 74 (1993) 2818.

[4] K. Allakhverdiev, N. Akhmedov, Z. Ibragimov, S. Ellialtioglu, K. Lothar, D. Haarer, Solid State Commun. 93 (1995) 147.

[5] N.B. Singh, D.R. Suhre, V. Balakrishna, M. Marable, R. Meyer, N.C. Fernelius, F.K. Hopkins, D.E. Zelmon, Prog. Cryst. Growth Charact. 37 (1998) 47.

[6] R. Bube, Photoelectronic Properties of Semiconductors, Cambridge University, Cambridge, 1992.

[7] V.I. Chmyrev, V.M. Skorikov, E.V. Larina, V.V. Zuev, Inorganic Mater. 38 (2002) 751.

[8] G. Micocci, A. Rizzo, A. Tepore, J. Appl. Phys. 58 (1985) 1274.

[9] A. Aydinli, N.M. Gasanly, K. Goksen, Mater. Res. Bull. 36 (2001) 1823.

[10] A.F. Qasrawi, N.M. Gasanly, Cryst. Res. Technol. 37 (2002) 587.

[11] R. Chen, Y. Kirsh, Analysis of Thermally Stimulated Processes, Pergamon Press, Oxford, 1981, p. 9.

[12] C. Micocci, A. Rizzo, A. Tepore, J. Appl. Phys. 54 (1983) 1924. 